\title{
Hyperbolicity Outside a Compact Set and Homogeneous Spaces $\left(^{*}\right)$.
}

\author{
Givliana Gigante
}

Summary. - We study complex manifolds, which satisfy the following property: there exists a compact $K$ such that $M-K$ is Kobayashi-hyperbolic. In section 2 , we study in some detail the case when $M$ is homogeneous under the action of a complex Lie group. In section 3, we give some results when $M$ is homogeneous under the action of a real Lie group of holomorphic transformations.

\section{Introduction.}

The first part of this paper is devoted to prove that if $M$ is a complex manifold such that for some compact subset $K, M-K$ is a Kobayashi-hyperbolic manifold (which we will refer to as *-property), then the group of the automorphisms of $M$ is a Lie group, that is, for a well known theorem of Bochner and Montgomery (see [Bo-M]) it is a locally compact group. This result is already known in the case when $M-K$ is a Riemann domain over a bounded domain of $C^{n}([\mathrm{Fu}])$. The *-property occurs, for example, in the blow-up of an hyperbolic manifold along a compact submanifold. Some results are also given on the behavior of the infinitesimal automorphisms of $M$ with respect to the complex structure $J$ of $M$.

In the second part of the paper we deal with manifolds with the *-property, under the assumption that they are homogeneous under the action of a complex Lie group. In some sense, this problem could be viewed as a generalization of the characterization of complex homogeneous strongly pseudoconcave manifolds, done in ([H-Sn 1]) by Huckleberry and Snow; there it is used a famous result ([A-H]), about the equivalence between analytic and algebraic dependence of meromorphic functions, that we do not have. Anyway, some considerations of ([H-Sn1]) can be proven in a simpler way by using *-property; although we don't obtain a classification's list. Among other results, we prove that manifolds with *-property which are complex homogeneous are, besides the compact or hyperbolic ones, holomorphic fiber bundles over compact complex homogeneous manifolds with 1-dimensional fiber.

The object of the third part of the paper are complex manifolds with the *-property,

(*) Entrata in Redazione il 15 luglio 1997.

Indirizzo dell'A.: Dipartimento di Matematica, Via M. D’Azeglio, 83, Università di Parma, Parma (Italia). 
which are homogeneous under the action of a real Lie group of holomorphic transformations. Theorem 2.3 deals with the case when the group is semisimple of first category; other results are gathered together about holomorphic functions theory on these manifolds. Let's begin with some notation:

$\Delta_{h}=\{z \in C:|z|<h\}, \Delta_{1}=\Delta ;$

$B_{\mu}(x, r)$ is the ball of center $x$ and ray $r$ with respect to the distance $\mu$;

$\varrho$ is the distance of Poincare in $\Delta$;

Aut $M$ is the group of biholomorphic automorphisms of $M$;

$J$ denotes the complex structure of the real tangent space of $M$;

bd.ry denotes the topological boundary.

\section{1. - The *-property.}

In this section we will consider complex manifolds $M$, that have the following property:

*-property: there exists a compact subset $K$ of $M$ such that $M-K$ is hyperbolic.

THEOREM 1.1. - Let $M$ be a complex manifold with the *-property. Then, the group of biholomorphic automorphisms of $M$ is a Lie group.

Proof. - Let's divide the proof in three steps:

First step: let $\mathrm{R}$ be a compact subset of $M$ such that $M-R$ is hyperbolic. We build a symmetric neighborhood $U$ of the identity in Aut $M$ with the following properties: there are three domains $D^{\prime} \subset \Delta \subset c D^{\prime \prime}$ with $\operatorname{R} \subset D^{\prime}$ such that for any $f$ in $U$ we have:

$$
f(R) \subset \bar{D}^{\prime}, \quad f(D) \subset D^{\prime \prime},
$$

and such that every sequence $f_{n}$ has a subsequence that converges uniformly on compacts of $D$ to $f: D \rightarrow D^{\prime \prime}$, with $f(\mathrm{R}) \subset D$; while the sequence $f_{n}^{-1}$ converges uniformly on compacts in $D$ to $g: D \rightarrow D^{\prime \prime}, g(\mathrm{R}) \subset D$; in particular, we get also that fog is defined over $\mathrm{R}$ where it coincides with the identity and then $f$ is iniective on $D$. We can verify in the proceeding of the proof of this first step that we can take as domain $D$ an arbitrary small neighborhood $\mathrm{R}$.

For every $x \in \mathbb{R}$, let's take neighborhoods $U_{x} \Subset \subset V_{x} \Subset \subset U_{x}^{\prime} \Subset \subset V_{x}^{\prime}$ and let $D=$ $=\bigcup_{i=1, \ldots, r} U_{x_{i}}$ in such a way that it contains R and let's choose an open $D^{\prime} \lessdot \subset D$, which still contains R, moreover let be $D^{\prime \prime}=\bigcup_{i=1, \ldots, r} U_{x_{i}}^{\prime}$. We can suppose that the $V_{x_{i}}^{\prime \prime}$ 's are hyperbolic.

Now, for every $i=1, \ldots, r$, we consider

$$
\mathbb{W}\left(\left(\bar{U}_{x_{i}}, V_{x_{i}}\right)=\left\{f \in \operatorname{Aut} M \cdot f\left(\bar{U}_{x_{i}}\right) \subset V_{x_{i}}\right\}\right.
$$


and

$$
\mathbb{W}\left(\left(\bar{U}_{x_{i}}^{\prime}, V_{x_{i}}^{\prime}\right)^{-1}=\left\{f^{-1}: f\left(\left(\bar{U}_{x_{i}}^{\prime}\right) \subset V_{x_{i}}^{\prime}\right\} .\right.\right.
$$

Let $U^{\prime}=\bigcap_{i=1, \ldots, r} \mathbb{W}\left(\left(\bar{U}_{x_{i}}, V_{x_{i}}\right) \cap \mathbb{W}\left(\left(\bar{U}_{x_{i}}^{\prime}, V_{x_{i}}^{\prime}\right)^{-1} \cap \mathbb{W}\left(\mathrm{R}, D^{\prime}\right)\right.\right.$ and $U=U^{\prime} \cap U^{\prime-1}$.

Let $f_{n} \subset U$, then the $f_{n}^{\prime}$ 's, if restricted to $U_{x_{i}}$, take their values in $V_{x_{i}}^{\prime}$; so we have an equicontinuous family with respect to the Kobayashi's distances of $U_{x_{i}}$ and of $V_{x_{i}}^{\prime}$, furthermore $f_{n}(x)$ are all in the compact $V_{x_{i}}$ and then there is a subsequence uniformely convergent on compacts of $U_{x_{i}}$. It is clear now how to extract from $f_{n}$ a subsequence, which converges uniformely on compacts in $D$ to a function $f: D \rightarrow D^{\prime \prime}$; of course we get also $f(R) \subset \bar{D}^{\prime} \subset D$.

If we apply the same arguments to $f_{n}^{-1}$ we get the convergence of some subsequence to $g: D \rightarrow D^{\prime \prime}$, with $g(\mathrm{R}) \subset D$. Let's only observe that, since $f_{n}^{-1}\left(\left(\bar{U}_{x_{i}}\right) \subset V_{x_{i}}^{\prime}\right.$ we can obtain a subsequence that converges on the compacts of $D^{\prime \prime}$, so $g$ is defined on $D^{\prime \prime}$ and $g$ of is on $D$ the identity, concluding that $f$ is injective on $D$.

Second step: we choose a compact $\mathrm{R}$, connected, of $M$ which contains $K$ in its interior. Denote by $\mu$ the Kobayashi's distance of $M-K$. Now, let $r$ be such that

$$
\{x \in M-K: \mu(x, \text { bd.ry } R)<2 r\}
$$

is relatively compact in $M$ and let $D$ be a neighborhood of R, relatively compact in $M$ and such that

$$
\mu((\text { bd.ry } D \text {,bd.ryR }) \leqslant r \text {. }
$$

Denote by $\beta$ the Kobayashi's distance of $M-\bar{D}$.

Let's now prove that there exists a constant $k>0$ such that, for every $x \in M-\bar{D}$ such that $\mu(x$,bd.ry $D)>r$, there is $\varepsilon(<r$ and dependent by $x$ ) so that, being $B$ the balls for the Kobayashi's distance,

$$
B_{\beta}(x, \varepsilon) \subset B_{\mu}(x, \varepsilon) \subset B_{\beta}(x, k \varepsilon) .
$$

Let $x, y$ be two points in $M-\bar{D}$ such that

$$
\mu(x, \text { bd.ry } D)>r \quad \text { and } \quad \mu(y \text {, bd.ry } D)>r \quad \text { and } \quad \mu(x, y)=s<r / 2
$$

and let $s$ be so small that every point of $B_{\mu}(x, 2 s)$ has a distance greater of $r$ from the boundary of $D$.

Let $f_{i}: \Delta \rightarrow M-K, i=1, \ldots, m$, be a chain of holomorphic functions by means of which we can approximate

$$
\begin{gathered}
\mu(x, y), \quad \text { that is } f_{i}(0)=x_{i}, f_{i}\left(a_{i}\right)=x_{i+1} ; \\
x_{1}=x, x_{m+1}=y \quad \text { and } \quad s-1 / n<\sum_{i} \varrho\left(0, a_{i}\right)<s .
\end{gathered}
$$

Let's note that $\mu\left(x_{i}\right.$, bd.ry $\left.D\right)>r$, for every $i$.

If, for every $i$, we have $f_{i}(\Delta) \subset M-\bar{D}$, then this same chain can be used to compute $\beta(x, y)$, therefore we get $s-1 / n<\beta(x, y)<s$; suppose instead that $f_{1}(\Delta)$ intersects $\bar{D}$, and let $a_{1}$ in $\Delta$ such that $f_{1}\left(a_{1}\right)=x_{2}$ and $\varrho\left(0, a_{1}\right)<s<r / 2$, then we choose $h$ in such a 
way that:

$$
\left|a_{1}\right| / h<1, \quad h<1, \quad f_{1}\left(\Delta_{h}\right) \cap \bar{D}=\emptyset .
$$

Let's continue this operation of "reduction", when it is necessary, along all the analytic chain, keeping in mind that we can take an unique $h$, that does the work along all the chain, for example $h=\left(e^{r}-1\right) /\left(e^{r}+1\right)$. Now, consider the chain given by $g_{i}(z)=$ $=f_{i}(h z)$; it is a chain in $M-K$ which joins $x$ with $y$, from which we obtain that $\beta(x, y)<\sum_{i} \varrho\left(0, a_{i} / h\right)$.

Observing that the function $\log (1+t / h) /(1-t / h)$ behaves as $(1 / 2 h) \cdot \log (1+t) /$ $/(1-t)$ when $t \in \mathbb{R}_{+}, t \rightarrow 0$ we have that $\beta(x, y)$ is less than $(1 / 2 h) \mu(x, y)$ if $\mu(x, y)=s$ is small enough.

It needs to note also that, while $s$ depends on $x$, we get that $h$ does not depends on $x$, but only on $r$. Therefore, for every $x \in M-\bar{D}$, with $\mu(x$, bd.ry $D)>r$, there is $\varepsilon>0$ such that

$$
B_{\mu}(x, \varepsilon) \subset B_{\beta}(x,(1 / 2 h) \varepsilon) .
$$

The fact that $B_{\beta}(x, \varepsilon) \subset B_{\mu}(x, \varepsilon)$ derives from usual properties of Kobayashi's distances.

Third step. Let $S$ be a compact which contains $\bar{D}$ and such that $\mu($ bd.ry $S$, bd.ry $D)>$ $>r$ (it exists by the way it has been chosen $r$, in step two). Moreover, we chose a relatively compact subset $T$ which contains $S$ in its interior and we build a symmetric neighborhood $V$ of the identity in Aut $M$, as in step one with $\mathrm{T}$ in place of $\mathrm{R}$. Now, we want to prove that the intersection of $U$ and $V$ is a locally compact neighborhood of the identity in Aut $M$.

Let $f_{n}$ be a sequence of automorphisms in $U \cap V$, we can suppose (by taking a subsequence, if it needs) that it converges uniformly on the compacts of $\mathbf{T}$ to a map which is injective in $\mathbb{T}$ and furthermore we know, again by step one, that the $f_{n}$ 's, with their inverses, carry $\mathrm{R}$ on $D$, therefore we get

$$
f_{n}: M-\bar{D} \rightarrow M-R \subset M-K .
$$

Let's prove now that, for every $x \in M-S$, there exists $V_{x} \subset M-S$ with the following property:

if there exists $y \in V_{x}$ such that $f_{n}(y)$ has some accumulation point, then $f_{n}$ converges uniformly on compacts of $V_{x}$. Let $\varepsilon>0$ be so small that 1 holds and that $B_{\beta}(x, k \varepsilon)$ is relatively compact in $M-\bar{D}$; and let $V_{x}=B_{\beta}(x,(k / 8) \varepsilon)$. Let $y$ in $V_{x}$ so that $f_{n}(y)$ has some accumulation point and let $n^{\prime}$ be such that for $n>n^{\prime}$ it is $\mu\left(f_{n}(y), f_{N}(y)\right)<(k / 8) \varepsilon$. We get

$$
\begin{aligned}
\mu\left(f_{n}(z), f_{N}(x)\right) & <\mu\left(f_{n}(z), f_{n}(y)\right)+\mu\left(f_{n}(y), f_{N}(y)\right)+\mu\left(f_{N}(y), f_{N}(x)\right)< \\
< & \beta(z, y)+\beta(x, y)+\mu\left(f_{n}(y), f_{N}(y)\right)<(k / 4) \varepsilon+(k / 8) \varepsilon+(k / 8) \varepsilon<(k / 2) \varepsilon .
\end{aligned}
$$


Therefore, if $n>N$ and $z \in V_{x}$, we have

$$
f_{n}(z) \in B_{\mu}\left(f_{N}(x),(k / 2) \varepsilon\right)<f_{N}\left(B_{\beta}((x,(k / 2) \varepsilon) \cup \bar{D})\right.
$$

which is relatively compact in $M$, and then $f_{n}(z)$ has some accumulation point in $B_{\mu}\left(f_{N}(x), k \varepsilon\right)$; the equicontinuity of the family of applications from $V_{x}$ to $B_{\mu}\left(f_{N}(x), k \varepsilon\right)$ with respect to the Kobayashi's distances gives a subsequence which converges uniformly on compacts of $V_{x}$ to an holomorphic map.

It is easy now, keeping in account the uniform convergence on compacts of $T$, to prove that $f_{n}$ has a subsequence which converges on compacts of $M$ to a map $f$; same arguments, applied to the sequence of the inverse maps, prove that $f$ is in Aut $M$.

REMARK 1.a. - Every strongly pseudoconcave manifold, that is a complex manifold (of dimension $n$ ) endowed with a smooth, non negative function $\varphi: M \rightarrow \mathrm{R}$ satisfying:

(i) $M_{c}=\{m \in M / \phi(m)>c\}$ is relatively compact in $M$ for $c>0$

(ii) for some $c>0$, the Hermitian form

$$
L(\varphi) m=\sum \frac{\bar{\partial} \partial \varphi_{(m)}}{\partial z_{i} \bar{\partial} z_{j}} d z_{i} \otimes \overline{d z_{j}}
$$

has $n$ positive eigenvalues on the complex tangent space $T_{c}(M)_{m}$, for all $m \notin M_{c}$, satisfies our property namely, when $c$ is large enough, $M-M_{c}$ is contained in a bounded domain of a Stein space (see [H-Sn1], [Fu]).

REMARK 1.b. - The *-property is not preserved from $M$ to some covering of $M$, unless the covering is finite. Viceversa, it is clear that if a covering of $M$ satisfies the property, then $M$ itself satisfies it (see [K2], for hyperbolicity and coverings).

REMARK 1.c. - If $M$ is biholomorphic to a product $M_{1} \times M_{2}$ of complex manifolds and has the *-property, then we have the following possibilities:

(i) $M$ is hyperbolic,

(ii) $M_{1}$ is non compact, while $M_{2}$ is compact and hyperbolic,

(iii) $M$ is compact.

Of course, we can suppose that $K$ is the product of two compacts $K_{1}$ of $M_{1}$ and $K_{2}$ of $M_{2}$. Now, if one of the factors, say $M_{1}$, is not compact the other one must be hyperbolic. Namely, let $p$ be a point of $M_{1}-K_{1}$, then the submanifold of $M-K$ given by $\{p\} \times M_{2}$ must be hyperbolic, so $M_{2}$ must be hyperbolic. Let's note that if $M$ is homogeneous, then the second case cannot occur.

REMARK 1.d. - We think that there are not open domains in $C^{n}$, which satisfy our property, when $n>1$ unless the hyperbolic ones. We aren't able at the moment to prove it, nevertheless we have the following result in this direction 
Proposition. - If $D$ is a convex domain in $C^{n}, n>1$ and satisfies the *-property, then $D$ is hyperbolic.

Proof. - We extend the proof of proposition 2.8 of [Fr] on convex hyperbolic domains to hold when $D$ is hyperbolic except for a compact set $K$. Namely, we will find complex coordinates $z_{1}, \ldots, z_{n}$ such that $D+B(0, r)$ has empty intersection with the coordinate hyperplanes; after that we can easily define an holomorphic and bounded embedding of $D$ in $C^{n}$, which obviously says that $D$ is hyperbolic ([K2]). The way is to find, by induction, $n$ complex hyperplanes, each one with distance from $D$ greater than $r$, which intersect transversally. By the convexity of $D$, we can find an hyperplane with distance $>r$ from $D$; now suppose we have already $k$ hyperplanes as above and let's find the $(k+1)$-th hyperplane. Let $L$ be a complex line contained in the intersection of the $k$ hyperplanes we have, then, through any $p \in D$ we take the line parallel to $L$. Since $D-K$ is hyperbolic, this line cannot be contained in $D-K$, then either it intersects $K$ in some point or it get out from $D$ intersecting the boundary of $D$.

Now, if all these lines, parallel to $L$, are contained in $D$, then $D$ can be written as union of parallel lines crossing $K$ which is impossible since $D$ is an open set. So there exists $p$, such that the line $L^{\prime}$ through $p$ has a point $q$ in common with the boundary of $D$. Let's consider the hyperplane $H$, which supports the convex $D$ at $q$; its intersection with $L^{\prime}$ is transversal and if we take a vector $v$, with $\|v\|>r$, orthogonal to $H$, the hyperplane $H+v$ does the work.

COROLLARY 2.1. - Let $M^{\prime}$ be a hyperbolic manifold and let $N$ be a complex compact submanifold of $M^{\prime}$. Let $M$ be the blowing-up with $N$ as center. Then Aut $M$ is a Lie group.

ProOF. - It is enough to remark that the operation of blowing-up consists of a proper map $\pi: M \rightarrow M^{\prime}$ which induces a biholomorphism between $M-\pi^{-1}(N)$ and $M^{\prime}-N$.

Let's remark now that the vector fields $X$ over $M$ which are the generators of global one parameter subgroup of holomorphic automorphisms of $M, t \rightarrow \exp t X$, constitute the Lie algebra $\alpha(M)$ of the group Aut $M$. Not all the infinitesimal automorphisms of the complex structure, characterized by the condition: $[X, J Y]=J([X, Y])$ for every vector field $Y$ on $M$, belong to $\alpha(M)$. It is interesting to know whether there are infinitesimal automorphisms $X$ in $\alpha(M)$ such that the infinitesimal automorphism $J X$ does not belong to $\alpha(M)$. In order to study this problem suppose that $X$ and $J X$ belong both to $\alpha(M)$. It follows that Aut $\mathrm{M}$ has a complex one parameter subgroup $H$ that is there is an homomorphism $\phi$ from $C$ to Aut $M$ such that, for every $p \in M, z \rightarrow \phi(z) p$ is an holomorphic map from $C$ to $M$. Since $M-K$ cannot contain any complex line, either such a map is constant and equal to $p$ or it takes some values in $K$; by that we can deduce that $M=$ $=H . K \cup F$, where $F$ is the set of the points which are left fixed by all the automorphisms which belong to $H$ and $H . K$ is the union of the orbits of $H$ through points of $K$. From this remark, we can deduce the following conclusions:

COROLLARY 3.1. - Let $K$ be nowhere dense in a submanifold (real) of $M$ with dimension $\leqslant 2 n-2$, if $X \neq 0$ belongs to $\alpha(M)$ then $J X$ does not belong to $\alpha(M)$. 
Proof. - The interior part of $H . K$ is empty, thus $F$ has a non empty interior, that implies that every element of $H$ fixes an open set and then it is the identity; it follows that $X=0$.

COROLLARY 4.1. - Suppose that there are bounded non constant functions on $M$. If $X \neq 0$ belong to $\alpha(M)$ then $J X$ does not belong to $\alpha(M)$.

Proof. - Let $f$ be bounded and non constant; thus, $f$ has to be constant along $H p$, for every fixed $p$ in $K$, so we get that $f(H . K)=f(K)$.

Since $F$ must have empty interior part, otherwise it would be $X=0$, then every $q \in$ $\in F$ can be view as limit of points of type $h_{m}\left(k_{m}\right)$; therefore $f(q)=\lim f\left(k_{m}\right)$, from which it follows that $f(M)=f(K)$ which is impossible, since $f$ must be open.

\section{2. - The complex homogeneous case.}

Let $M$ be a connected complex-homogeneous complex manifold, that is there exists a connected complex Lie group $G$ which acts transitively and effectively (the only element of $G$ which leaves every point of $M$ fixed is the identity), on $M$ by biholomorphic transformations. If we denote by $H$ the isotropy subgroup of a fixed point $m$, then there is a natural identification of $M$ and $G / H$, the right $H$-coset space of $G$. By the effectiveness, we can consider $G$ as a subgroup of Aut $M$. From now on, we will always write $G / H$ for $M$.

First of all, we want to remark that in the following, we can relax the condition: hyperbolic outside a compact subset in the following weaker condition, which we will again refer to as:

*-property: there exists a compact subset $K$ such that $M-K$ has no complex lines, that is any holomorphic map $f: C \rightarrow M-K$, is constant.

Let $L$ be a closed subgroup of $G$ which contains $H$, and let's consider the projection $p: G / H \rightarrow G / L$ with $L / H$ as fiber. Then we have the following

LEMMA 1.2. - If $K$ is a compact subset of $G / H$ such that $(G / H)-K$ is hyperbolic, then, we get the following possibilities: $L / H$ is discrete or $G / L$ is compact and $L / H$ has a finite number of connected components.

Proof. - If $p(K)$ is not all $G / L$ then $L / H$ must be hyperbolic since it is contained in an hyperbolic manifold. But that is impossible, since it is complex-homogeneous, unless $L / H$ has dimension zero and then it is discrete. If $p(K)$ is surjective then $G / L$ is obviously compact and $L / H$ inherits from $G / H$ the *-property.

Thus, $L / H$ must have a finite number of connected components.

REMARK 2.a. - In particular, the above lemma shows that, if $N$ denotes the normalizer of the connected component of the identity $H_{0}$, then either we get a covering $G / H \rightarrow$ $\rightarrow G / N$, or $G / N$ is compact (and in this case by a result of ([Bo-M]), $G / N$ will be a rational variety) and it holds for the fiber $N / H=N^{\prime} / J$ where $N^{\prime}=N / H_{0}$ is a Lie group and $J=$ $=H / H_{0}$ is discrete. 
We begin by studying the particular case when $M$ is a complex Lie group.

THEOREM 2.2. - Let $M$ be a complex Lie group with the *-property, then either $M$ is $C$, or $M$ is $C^{*}$, or $M$ is compact.

Proof. - It is known that every complex Lie group is biholomorphic, as complex space, to the space $G^{\prime} \times S$, where $G^{\prime}$ is a complex abelian Lie group without any non constant holomorphic function and $S$ is a Lie group which is a Stein manifold ([M-M]). Now, since $S$ cannot be compact and $G^{\prime}$ cannot be hyperbolic, we get either $M=S$ or $M=G^{\prime}$. Suppose $M=G^{\prime}$, then, by lemma 2 of ([Ba-O]), we have that $G^{\prime}$ is holomorphically isomorphic to $C^{n} / J$ where $J$ is a discrete subgroup of complex rank $n$. Moreover, if $G^{\prime}$ is non compact, that is $J$ has real rank less than $2 n$, then, if $n>1, G^{\prime}$ can be exausted by compacts whose boundaries are foliated by complex leaves, which cannot be hyperbolic complex manifolds since they are quotients of complex linear spaces $([\mathrm{A}-\mathrm{H}])$. Suppose now $M=S$. By ([M-M]), a complex Lie group is a Stein manifold if and only if it is biholomorphic to the space $C^{\prime} \times K^{c}$ where $K$ is a maximal compact subgroup of $S$ and $K^{c}$ is the complex Lie subgroup of $S$, which corresponds to the Lie algebra $t^{c}=t+$ $+i t, t$ being the real Lie algebra of $K$; moreover the intersection of $t$ and it must be zero. Again by Remark 1.c, we can deduce that either $S$ is biholomorphic to $C$ or to $K^{c}$. Let now be $M=K^{c}$, i.e. it is the complexification of one maximal compact subgroup. By a result of ([Sn3] p. 194), $K^{c}$ is isomorphic, up to finite central coverings, to the direct product of a complex semisimple Lie group and a toral factor, which is $\left(C^{*}\right)^{l}$. Applying again remarks $1 . c$ and $1 . b$, our problem can be reduced to prove that there are no semisimple Lie groups with our property.

Let's note that the proof of the theorem is completed when the dimension of $M$ is two, since there are no semisimple Lie groups of dimension two. Suppose now that we already proved the theorem for dimension less than $n$, and let's prove it for $n$. The complex semisimple Lie groups have holomorphic exact representations in linear spaces of finite dimension ([N-S], p. 529), therefore they are linear algebraic groups. Every parabolic subgroup $P$ of $M$ is connected and closed, since $M$ is complex (see p. 143 of [Hp]) and gives a fibration of $M \rightarrow M / P$ which has as fiber a connected complex Lie group with our property, but its dimension is strictly less than $n$, while $M / P$ is known to be compact ([Wa]). Since a parabolic subgroup cannot have dimension 1 , the proof of the theorem is completed.

REMARK ON KAEHLER HOMOGENEOUS MANIFOLDS $2 . b$. - The fundamental theorem in section 7.7 of [D-N 1] says that every homogeneous Kaehler manifold $M$ (i.e $M=G / H$ where $G$ is a connected real Lie group and $H$ is a closed subgroup, is a complex manifold endowed with a Kaehler $G$-invariant structure), is biholomorphic to the product of a bounded homogeneous domain $D$ and $C^{n} / J$ where $J$ is a discrete subgroup and a simply connected compact complex homogeneous manifold $M^{\prime}$. Now, by a remark of section 1 , we have the following possibilities for $M$ :

(i) $M$ is hyperbolic,

(ii) $M=C$ or $C^{*}$,

(iii) $M$ is compact. 
Namely, $M^{\prime}$ being complex, compact and homogeneous cannot be hyperbolic and then, if $M$ does not coincide with $M^{\prime}$, we get either $M=D \times\left(C^{n} / J\right)$ or $D \times\left(C^{n} / J\right)$ must be compact. In this last case, $M$ is compact, while if the first case occurs and $M$ is not $D$, then we have either $M=C^{n} / J$ or $C^{n} / J$ must be compact and hyperbolic. Now, the theorem above on groups completes the proof.

REMARK ON SOLVMANIFOLDS 2.c. - Let $M=G / H$, where $G$ is a solvable complex connected Lie group, satisfies the ${ }^{*}$-property. If $M$ is simply connected, then $M=C$.

Proof. - It is a simple matter to verify that we can suppose that $G$ is simply connected and $H$ is connected without any restriction. It is already known (see [O-R] p. 402) that $\mathrm{M}$ is biholomorphic to $C^{n}$, now the result follows obviously from *-property.

THE HOLOMORPHIC (AND MEROMORPHIC) REDUCTION MAPPING. - For an arbitrary complex space $X$ has been considered by Remmert, the equivalence relation:

$$
x_{1} \approx x_{2} \leftrightarrow f\left(x_{1}\right)=f\left(x_{2}\right) \quad \text { for all } f \in \mathcal{O}(X) .
$$

When $X=G / H$, where $G$ is a connected complex Lie group and $H$ is a closed complex subgroup then the space $X / \approx$ is Hausdorff and can be given a natural complex structure, and there exists a closed complex subgroup $L$ of $G$ containing $H$ so that $x_{1} \approx x_{2} \leftrightarrow$ $\leftrightarrow g\left(x_{1}\right)=g\left(x_{2}\right)$ where $g: G / H \rightarrow G / L$ is the natural projection ([G2]).

The homogeneous manifold $G / L$ is holomorphically separable and $g$ induces an algebra isomorphism $g^{*}: \mathcal{O}(G / L) \rightarrow \mathcal{O}(G / H)$. Note that in general we have $\mathcal{O}(L / H)$ different by $C$.

Just for the sake of completeness, we recall on this line the following result of ([G-H2]):

Let $g: G / H \rightarrow G / L$ be the holomorphic reduction bundle. If $G$ is nilpotent, then $G / L$ is a Stein manifold, the fiber $L / H$ is connected and $\mathcal{O}(L / J)=C$. The same result holds when $G$ is solvable and $H$ has a finite number of connected components ( see [H-O2]). But the fact that the base of the holomorphic reduction is Stein holds always when $G$ is solvable, ([H-O3])).

Applying the holomorphic reduction bundle to complex homogeneous manifolds with *-property, we get the following result:

either $L / J$ is discrete and $G / H$ is a covering of $G / L$, which is holomorphically separable or $J=G$, and $G / H$ has no holomorphic non constant functions.

The meromorphic reduction bundle is defined in the same way, using meromorphic instead of holomorphic functions.

THE HYPERSURFACES REDUCTION BUNDLE. - Let $\mathscr{N}(X)$ denote the set of all hypersurfaces in $X$, where $X=G / H$. Then there exists a closed complex subgroup $J$, containing $H$, such that $H^{0}$ is normal in $J$ and $J / H^{0}$ is a complex Lie group acting holomorphically on the fiber $J / H$ of the bundle projection $\mathfrak{h}: G / H \rightarrow G / J$ and $\mathfrak{h}(x)=\mathfrak{h}(y)$ iff $x$ is hypersurface-equivalent to $y$, that is $x$ belongs to a hypersurface $F$ if and only if also $y$ does; 
moreover $G / J$ is hypersurfaces-separable, i.e. if two points of $G / J$ are equivalent in the above sense then they coincide.

Let's remark that, if $G / H$ satisfies *-property, then

either $G / H$ is a bundle over $G / J$ which is a compact projective algebraic manifold ([Gr-R]) with a theoretically parallelizable fiber with positive dimension and with *property and, of course, a finite number of connected components

or $\operatorname{dim} H=\operatorname{dim} J$ and then $G / H$ is a Kaehler manifold. ([H]) covering of a Kaehler manifold $G / J$, which has *-property and is hypersurfaces-separable.

We quote the following result of ([Be-O]): since a complex homogeneous manifold under a semisimple complex Lie group is kaehler if and only if the isotropy subgroup is algebraic, thus if $G$ is a semisimple complex Lie group, then $J$ is exactly the Zariski-closure of $\mathrm{H}$. Moreover, in this case, the hypersurfaces reduction coincides with the meromorphic reduction.

In ([Sn2]), D. M. Snow introduces the following definition:

DEFINITION. - Let $G$ be a complex Lie group and $H$ a subgroup of $G$. We say that $H$ is a quasi-algebraic subgroup of $G$ if there is a holomorphic representation of $G$, $r: G \rightarrow G L(n, C)$ such that

$$
H=\varrho^{-1}\left(\varrho(H)^{*} \cap \varrho(G)\right) .
$$

Snow shows that $H$ is quasi-algebraic if and only if $G / H$ is $G$-equivariantly mapped in some projective space. Moreover, he defines the quasi-algebraic closure of a subgroup $H$ to be the smallest quasi-algebraic subgroup $H^{*}$ of $G$ which contains $H$ (this is the same as the intersection of all the quasi-algebraic subgroups of $G$ which contain $H$ ). An interesting property of the quasi-algebraic closure $H^{*}$ is the fact that it normalizes the connected component of the identity: $H^{0}$.

The following theorem of Snow on solvable groups will be very useful.

THEOREM. - Let $G$ be a connected solvable complex Lie group and let $H$ be a quasialgebraic subgroup of $G$. Then $G / H$ is biholomorphic to $\left(C^{*}\right)^{s} \times(C)^{t}$.

THEOREM 3.2. - If G/H is non compact and has *-property and $G$ is a solvable complex Lie group, then $G / H$ is a homogeneous fiber bundle over a compact solvmanifold whose fiber is a connected 1-dimensional homogeneous space.

Proof. - If $\mathrm{H}$ is a quasi-algebraic subgroup the result is evident by the theorem above. Otherwise, let $H^{*}$ be the quasi-algebraic closure of $H$. Then, again by Snow's theorem either $H^{*}=G$ or $\operatorname{dim} H=\operatorname{dim} H^{*}$.

In the last case, $G / H$ will be a covering of $G / H^{*}$, that is $G / H$ will be $C$ or $C^{*}$. In the first case, we will have $G / H=H^{*} / H=\left(H^{*} / H^{0}\right) /\left(H / H^{0}\right)$, so we restrict our attention to the case when the manifold is $N / \mathrm{J}$ with $N$ solvable and $J$ discrete. Furthermore, recall that when $N$ is abelian, $N / J$ will be a group and we already know the result. Now, we quote the following result in [H-04] Lemma 7 p. 187: 
LEMMA. - Let $N$ be a connected, non-abelian solvable complex Lie group and let $J$ be a diserete subgroup. Then, there is a proper, positive dimensional closed subgroup $Y$ of $N$ which contains $J$.

We prove the following:

Lemma 4.2. - Let $N / J$ be non compact, $N$ be a solvable complex Lie group and $J$ be a closed discrete subgroup. If N/J satisfies the ${ }^{*}$-property, then there exists a closed complex 1-dimensional subgroup of $N$ containing $J$.

Proof. - If $N$ is an abelian Lie group, we already know that $N / J$ must be $C$ or $C^{*}$. So, let $N$ be non abelian; applying the above lemma, we get the projection $N / J \rightarrow N / Y$, this last being compact, with fiber $Y / J$ of less dimension than $N / J$, which can be taken connected, simply substituting $Y$ by $Y^{0} J$. Now the fiber inherits the *-property and it is non compact. So we get a one dimensional, closed subgroup of $Y$ which contains J. Now the proof of theorem 3.2 is quite done.

REMARK 2.d. - If we look for manifolds which are locally separable by global hypersurfaces (that is any point is isolated in the intersection of a finite number of global hypersurfaces.: loc.sep.hyp) then we can use the following result of [O-R]

LEMmA. - Let $M$ be a complex solvmanifold loc. sep. hyp. and suppose that there are no non constant holomorphic functions on $M$, then $M$ is a Cousin group.

We recall that a Cousin group is a group of type $C^{n} / Y$, where $Y$ is a discrete subgroup, and we already know groups which have *-property; they are $C$ or $C^{*}$ when they are non compact.

Moreover, in the same paper, the authors prove that, in the hypothesis of the lemma, the holomorphic reduction of $G / H$ has a Stein manifold as base and a Cousin group as fiber. Therefore, we get the following

Corollary. - Let $G / H$ be loc. sep. hyp. and satisfy *-property. Let $G$ be solvable. If $G / H$ is noncompact, then $G / H$ is Stein.

Proof. - What we need is just to consider the fact that, either $G / H$ is a Cousin group or $G / H$ is holomorphically separable. In this last case, it must be a covering of a Stein manifold, and therefore it is a Stein manifold.

Let's concentrate to the case when $G$ is a reductive non abelian, complex Lie group.

THEOREM 5.2. - Let $G / H$ satisfy *-property, and let $G$ be a reductive complex Lie group. If $G / H$ is holomorphically separable, then $G / H$ is a bundle over a flag manifold with fiber $C^{*}$ or $C$.

Proof. - Let's begin reporting a theorem, which can be found in ([A2], p. 220). When $G$ is a complex reductive Lie group and $H$ is a closed subgroup, the following conditions are equivalent: 
(i) $G / H$ is holomorphically separable.

(ii) $H$ is closed in the Zariski topology and there exist a holomorphic finite-dimensional representation $\pi: G \rightarrow G L(W)$ and a vector $w \in W$ such that

(a) $P=g \in G / \pi(g) w \in C w\}$ is a parabolic subgroup;

(b) $H \subseteq Q$, where $Q$ is the subgroup of codimension 1 in $P$, defined by the condition $g \in Q \leftrightarrow \pi(g) w=w$;

(c) the unipotent radical of the linear algebraic group $H$ is contained in the unipotent radical of $Q$.

In our hypothesis, the above theorem says that $H$ is Zariski-closed and we get projections $G / H \rightarrow G / Q \rightarrow G / P$ where $P / Q$ is biholomorphic to $C^{*}$ by $g Q \rightarrow \pi(g) w / w$ for $g \in P$ (namely $g \in P \rightarrow \pi(g) w / w$ is a character of $P$ which has $Q$ as kernel). So, $P / Q$ cannot be compact, that implies that $G / Q$ cannot be compact, and then $H$ must have codimension 1 in $P$; so we get a bundle projection $G / H \rightarrow G / P$ over the flag manifold $P$ whose fiber, being $P$ connected, is $C$ or $C^{*}$.

THEOREM 6.2. - If G is semisimple and G/H satisfies *-property, then $G / H$ cannot be Stein.

Proof. - By Matsushima ([Ma1,2]), $H$ must be reductive, so its intersection with $R_{u}$, the unipotent radical of $P$, must have dimension zero. Since its codimension in $P$ is one then $R_{u}$ should have dimension $\leqslant 1$.

Moreover, $H$ cannot contain the borelian of $P$ and $H$ should be invariant in $P$ by the fact that $H=Q$, kernel of a character of $P$. So the Lie algebra of $P$ should be the direct product of the ideals given by the Lie algebras of $H$ and $R_{u}$, thus this last one would be in the center of the algebra, which is obviously absurd.

REMARK 2.e. - If $G$ is semisimple, and $G / H$, with *-property, is meromorphically separable, then $G / H$ is Kaehler and $H$ is Zariski closed [Be-O]. In particular, if $H$ is discrete, then it must be finite and then $G / H$ cannot satisfy *-property.

THEOREM 7.2 (The algebraic case). - Let $G$ be a linear algebraic group and $H$ be a $Z$ ariski closed subgroup of $G$. Let $G / H$ be a non compact manifold with the ${ }^{*}$-property. Then, $G / H$ is a bundle over a compact manifold whose fiber is connected and 1 dimensional.

Proof. - Since the radical $R$ of $G$ is algebraic, then $R H$ is a Zariski closed subgroup of $G$ which contains $H$. So we get the following possibilities:

(i) $\operatorname{dim} R H=\operatorname{dim} H$, which implies that $H$ contains $R$ (since $R$ is connected), and we can reduce ourselves to the case when $G$ is semisimple

(ii) $G / H \rightarrow G / R H, G / R H$ is compact and the fiber is a solvmanifold with *-property; in this case $R H / H$ is biholomorphic to $R / R \cap H$, which is a solvmanifold with *-property, so there exists a closed subgroup of $R H$ which contains $H$ as a subgroup of codimension 1. This implies that $H$ has codimension 1 in a closed subgroup $L$ of $G$. That is 
$G / H \rightarrow G / L$ where $G / L$ is, of course compact, and $L / H$ has dimension 1 (in order to get the connectness of $L / H$, we can take $L^{0} H$ instead of $L$ ).

Now, it remains to study the case when $G$ is semisimple, and $G / H$ has no holomorphic non constant functions.

If $H^{0}$ is reductive, then $G / H^{0}$ is Stein ([M1]) and then $G / H$ would have holomorphic functions being the fiber $H / H^{0}$ finite. Since $H$ cannot be parabolic $(G / H$ would be compact, in this case), then $H$ cannot be a maximal subgroup of $G$ (see [Hp]).

If there exists a proper closed subgroup of $G$ of dimension greater then $H$ and containing $H$, then we can argue by inductive hypothesis to find that $H$ has codimension 1 in a subgroup of $G$. So, let $H$ have "maximal dimension". Now the subgroup $R_{u}(H)$ of all unipotent elements in the radical of $H$ is positive dimensional (see ([Hp]). Let $U=$ $=\left(R_{u}(H)\right)^{0}=\left(R_{u}\left((H)^{0}\right)\right)^{0}$; we get that $H$ is in the normalizer of $U$ in $G: N(U)$. Now, $N(U)$ is a Zariski closed subgroup of $G$, proper, since semisimple Lie groups cannot have non-trivial normal unipotent subgroup. So $N(U)$ has the same dimension as $H$, and $N(U)$ is a maximal subgroup of $G$. Namely, if $L \supset N(U)$, then it must be $\operatorname{dim} L=$ $=\operatorname{dim} N(U)$ and then $L^{0}=N(U)^{0}$ which implies

$$
\left(\left(R_{u}(L)\right)^{0}=\left(R_{u}\left((L)^{0}\right)\right)^{0}=\left(R_{u}\left((N(U))^{0}\right)\right)^{0}=\left(R_{u}(N(U))\right)^{0}\right.
$$

and then $L=N(U)$. So $N(U)$ should be parabolic (and connected) which is absurd. So, if $G$ is semisimple, there must be a closed subgroup $P$ of $G$, where $H$ has codimension 1, and $G / P$ is, of course, compact.

COROLLARY 8.2. - If $G$ is a semisimple group and $G / H$ is meromorphically separable and satisfies *-property, then $\mathrm{G} / \mathrm{H}$ is a bundle over a flag manifold whose fiber has dimension 1.

Proof. - By ([Be-0]) $H$ must be Zarisky-closed, so the hypothesis imply that we are in the algebraic case.

CoRolLaRY 9.2. - If $G / H$ is Stein and satisfies *-property, then $G / H$ is a solvmanifold.

Proof. - The semisimple part $S$ of $G$ must have some closed orbit in $G / H$ which is Stein. ([R] and [Sn]). This orbit is of type $S / S \cap H$ and it has *-property. So, by theorem 7.2 , it must be $S \subset H$, which implies that $G / H$ is a solvmanifold.

Let's now suppose that the action of $G$ on $G / H$ is effective (we can always reduce ourselves to this case without loss of generality) and return to Snow's notion of quasialgebraic closure $H^{*}$ of a closed subgroup $H$ of a complex Lie group $G$. We get the following two cases:

(1) $\operatorname{dim} H=\operatorname{dim} H^{*}: G / H$ is a covering of $G / H^{*}$;

(2) $\operatorname{dim} H<\operatorname{dim} H^{*} \leqslant \operatorname{dim} G$ : we get a bundle $G / H \rightarrow G / H^{*}$, with compact base, whose fiber $H^{*} / H$, which has dimension less than $G / H$, satisfies *-property. Note that $H^{*} / H$ can be written as $N / J$, where $N$ is a complex Lie group and $J$ is discrete. 
Let's consider first the case when $H$ is a quasi-algebraic subgroup of $G$.

Following Snow [Sn2] p. 209, we realize $G / H$ as $\varrho(G) / \varrho(H)$ where $\varrho$ is a holomorphic representation $\varrho: G \rightarrow G L(n, C)$, for some $n$, such that $H=\varrho^{-1}\left(\varrho(H)^{*} \cap \varrho(G)\right)$ and consider the fact that the commutator subgroup $\varrho(R)^{\prime}=\varrho\left(R^{\prime}\right)$ is an algebraic unipotent subgroup of the linear group (see Snow, p. 210). Therefore, the subgroup $\varrho\left(R^{\prime}\right)\left(\varrho(H)^{*} \cap \varrho(G)\right)$ is a closed subgroup of $\varrho(G)$. So, we are now in the following situation: we get a subgroup $G$ of the linear group, a subgroup $H$ of $G$, which is the intersection with $G$ of a Zariski closed subgroup of the linear group. Moreover, $R^{\prime} H$ is closed and contains $H$. Note that we still get the effectiveness of the action of $G$ on $G / H$. Now, we get the following cases

(i) $G / H \rightarrow G / R^{\prime} H$, the base is compact and the fiber is a solvable manifold with *-property, then we know already that there exists a subgroup of $R^{\prime} H$ which contains $H$ as a subgroup of codimension one.

(ii) $\operatorname{dim} R^{\prime} H=\operatorname{dim} \mathrm{H}$, then $H$ contains the connected component of $R^{\prime}$; but then we can reduce ourselves to the case when $R^{\prime}$ is discrete, so $R$ has to be abelian.

Since $R$ is a reductive complex Lie group, then it has a unique structure of algebraic linear group which is compatible with its structure of complex Lie group, moreover, any holomorphic representation of $R$ must be rational (see [H-O1], or Hochschild-Mostow on Ann. of Math. 70, 1959). Let's recall that Snow shows that the quasi algebraicity of $H$ corresponds exactly to the fact that the manifold $M=G / H$ can be $G$-equivariantly mapped into some projective space $P^{n}$ (see Lemma 3.2 of [Sn2], p. 209). In effect, $M$ injects holomorphically and $G$-equivariantly in $M^{*}=G^{*} / H^{*}$, where $G^{*}$ is the algebraic closure of $G$ in $G L(n, C)$.

Now, $R$ is an algebraic subgroup of $G^{*}$, which implies that the action of $R$ on $M^{*}$ must have some closed orbits (see [Hp]). But $R$ acts also on $M$, and we can observe the following things:

an orbit of $R$ on $M$ is also an orbit of $R$ in $M^{*}$;

if an orbit of $R$ in $M^{*}$ intersects $M$, then it is contained in $X$;

the closure of an orbit of $R$ in $M$ is contained in its Zariski closure in $M^{*}$;

the closure of an orbit of $R$ in $M$ is contained in an union of orbits of $R$ in $M$, these orbits are algebraic variety of $M^{*}$ and have dimension less than the dimension of the orbit.

So, if $M$ itself does not coincide with an orbit of $R$ (that is we are in the solvable case) or if $G$ is semisimple (and thus, we are in the algebraic case), then there must be an orbit of $R$ which is closed in $M$. Let's denote by $L$ the subgroup of isotropy of $G$ at a point $y$ of this orbit, of course we can suppose that our subgroup $H$ at the beginning was $L$. Thus $R H$ is closed in $G$, since the orbit of $R$ is closed. Now, either $R H=G$ and $M$ is a solvmanifold, or $R H$ has the same dimension of $H$ (thus $H$ contains the radical) and we reduce to the semisimple case, otherwise $R H$ has a closed subgroup, which contains $H$ as subgroup of codimension one. If $H$ is not quasi algebraic, then as we already says at the case 2, we get a bundle fibration whose base is compact and with a fiber $H^{*} / H$ which is a manifold of our type, but of dimension less than $G / H$. So, simply by induction 
hypothesis, we get a subgroup of $G$ which contains $H$ as subgroup of codimension one.

So we have proven the following:

THEOREM 10.2 (the general case). - Let $G / H$ be non compact and satisfy the *-property. Then, we get a bundle projection $G / H \rightarrow G / L$, the base $G / L$ is a compact manifold, and the fiber is $C$ or $C^{*}$.

FinAL REMARKS FOR THE COMPLEX HOMOGENEOUS CASE 2.d. - Following ([H-Sn1]) at pag. 40, we observe that when $M$ is a bundle with $C^{*}$ as fiber, it can occur that $M$ is not a principal bundle, nevertheless it is covered in a 2 -to-1 way by a principal $C^{*}$-bundle: $M^{*}$. Thus, since it is a finite covering, also $M^{*}$ has *-property. So we can restrict ourselves to the case of principal $C^{*}$-bundles.

Moreover, the bundle $M^{*}$ is defined by transition functions with values in $C^{*}$, which define an (associated) holomorphic line bundle $L$ and $M^{*}$ can be viewed as the complement of the zero section in $L$.

When the base of this bundle is a homogeneous rational manifold, that is it is $S / P$ (a flag manifold) with $S$ complex and semisimple, then the $S$ action on the flag base can be lifted to a holomorphic $S$-action on $M^{*}$, moreover, since the bundle cannot be trivial, then $S$ acts transitively on $M^{*}$ and we get $M^{*}=S / H$. It turns out that $M^{*}$ is holomorphically separable iff the associated line bundle $L$ or its dual bundle $L^{*}$ is positive (see for all this [H-O1]).

In the recent years, there has been a great work around the theory of ends of homogeneous spaces (see [G1], [G-H1]; [Ak3]) The main result in [G1] gives the following:

if $G / H$ satisfies *-property and it is holomorphically separable, then $G / H$ has either one end or two and $G / H$ has two ends precisely if it is a homogeneous $C^{*}$-bundle over a homogeneous rational manifold whose associated line bundle is very ample. More precisely, $G / H$ is an affine homogeneous cone minus its vertex. (see theor. 5 of [G1]).

Furthermore, when $G / H$ is a bundle with fiber $C$ over the compact manifold $G / L$, then we get an affine bundle and we can consider the projective $P^{1}$-bundle $P$ obtained as usual by replacing $C$ with $P^{1}$. We get a holomorphic injection of $M=G / H$ in $P$, which preserves the bundle structure,

$$
(u, z) \rightarrow(u,[z, 1])
$$

where $[z, 1]$ are the homogeneous coordinates on the projective fiber. So $M$ can be seen as the complementary open subset in $P$ of the infinite holomorphic section s: $G / L \rightarrow P$, given by

$$
y \rightarrow(y,[1,0]) .
$$

Now, we are in the situation of an almost homogeneous compact manifold $P, M$ is the open orbit and the complementary analytic space $E$ is the base manifold $G / L$, which has codimension 1.

(This situation has been extensively studied in [H-O1], Chapter II). 


\section{3. - The (not necessarily complex) homogeneous case.}

THE ANTICANONICAL REDUCTION. - Let $G$ be a connected real Lie group acting almost effectively and transitively as a group of holomorphic transformations on the complex manifold $M=G / H$. Let $N$ be, as usual, the normalizer of $H^{0}$, let $J$ be the subgroup of $N$ given by

$$
J=\left\{k \in N: R(k): G / H^{0} \rightarrow G / H^{0}, g H^{0} \rightarrow g k H^{0} \text { is holomorphic }\right\}
$$

where $G / H^{0}$ is endowed with the complex structure induced by the covering $G / H^{0} \rightarrow G / H$.

Then $J / H^{0}$ is a complex Lie group and $G / H^{0} \rightarrow G / J$ is a holomorphic $J / H^{0}$-principal fiber bundle, the fibering $G / H \rightarrow G / J$ is locally holomorphically trivial. Moreover, the base of the anticanonical reduction $G / J$ is a $G$-orbit in a projective space. then

When $M$ has the *-property, since $J / H$ cannot be hyperbolic unless discrete,

either $M$ is a holomorphic fiber bundle over a compact projective manifold with a complex parallelisable manifold with *-property as fiber $J^{0}=H^{0}$

or the canonical reduction is a covering of a $G$-orbit in a projective space, that is

REMARK 3.a. - When $G$ and $H$ are complex Lie groups, then $J=N$ (see for the anticanonical reduction p. 61, [H-O1]).

REMARK 3.b. - Note the following result of [H.01] p. 65: when $G$ is solvable, and $M$ has no non constant holomorphic functions, then $G=J$, so that $G$ is a complex Lie group, $H$ is discrete and the action of $G$ on $M$ is holomorphic.

Let's describe the situation:

if $\mathcal{H}$ denotes the Lie algebra of $H$, then the Lie algebra of $J$ is given by

$$
\mathfrak{y}=\{X \in \mathcal{G}: \mathfrak{J}[X, Y]-[X, \mathfrak{J} Y] \in \mathcal{X} \text { for all } Y \text { in } \mathcal{S}\}
$$

$\mathfrak{I}$ is stable with respect to $\mathcal{I}$ and it coincides with $\mathcal{G} \cap \mathcal{N}(\mathfrak{q}$ ) (see $[\mathrm{K}]$, Lemma 1 ), where $\mathcal{G}$ is the Lie algebra of $G, \mathfrak{q}$ denotes the complex algebra in the complexified algebra $\mathcal{G}^{c}$ which defines the complex structure $y$ and

$$
\mathcal{N}(q)=\left\{X \in \mathcal{S}^{c},[X, q] \subset \mathfrak{q}\right\} ;
$$

now, since we get $\mathfrak{J}=\mathscr{C}$ arguing as in [Ha] and in $[\mathrm{K}]$, we get $\mathcal{N}(\mathfrak{q})=\mathfrak{q}$ and moreover it holds the following: the group $G$ acts on the grassmann manifold of all complex subspaces of dimension $m$ in $\mathcal{S}^{c}$, here $m$ is the complex dimension of $\mathfrak{q}$, via its adjoint representation Ad.

Furthermore, if $G^{c}$ is the complex subgroup of $G L\left(\mathcal{G}^{c}, C\right)$ corresponding to the subalgebra ad $\mathfrak{S}^{c}$ of $\mathcal{G} \mathfrak{L}\left(\mathfrak{G}^{c}, C\right)$, then one gets that $G / H$ is $G$-equivariantly immersed as an open complex submanifold in the complex homogeneous space $G^{c} / Q$ where $Q$ is the isotropy group at the point $\mathfrak{q}$, the Lie algebra of $Q$ being ad $q$ (this case occurs for 
example when $M$ is a bounded domain of $C^{n}$, ( $M$ hyperbolic), or in the pseudoconcave case, see [H-Sn], for $M=P^{n}-\overline{B^{n}}$ )

THEOREM 1.3. - If $M=G / H$ has more than one end, has the *-property and has some non constant holomorphic function, then $M$ is an affine homogeneous cone over a projective rational manifold with the vertex removed (see, the sections of remarks on complex homogeneous case).

Proof. - Applying theorem 10 of pg. 78 of [H-01], we get:

$G / H$ is a holomorphic fiber bundle with the complex parallelisable manifold $J / H$ as fiber over a base $G / J$ which is a projective rational manifold and $J / H$ has the same number of ends of $G / H$ and it has *-property. If there is some non constant function in $G / H$, then there must be some non constant holomorphic functions also on $J / H$, since the base is compact. But theorem 6 of [G1], p. 551, applied to the complex homogeneous manifold $J / H$, says that the $J / H$ (and then $G / H$ ) has exactly two ends and that $J / H$ is a bundle with connected, complex compact fiber over an affine homogeneous cone minus its vertex (for this result, it is used the holomorphic reduction). Now, since $J / H$ has the *-property then the only possibility is that $J / H$ itself is the affine cone and then, by theor 4 of [G1] $\mathrm{J} / \mathrm{H}$ must be exactly $C^{*}$. Since $G / H$ is a non trivial holomorphic $C^{*}$-bundle over the projective rational manifold $G / J$, the universal covering of the complex group Aut $(G / J)^{0}$ acts holomorphically and transitively on $G / H$, and we are in the case of complex homogeneous spaces (see also p. 80 [H-O1] and theorem 5 of [G1]).

In ([D-Z2]), the authors studied pseudokaehler manifolds admitting an effective, transitive and reductive group $G$ of automorphisms (pseudokaehler means that there is a Kaehler 2-form which is only non degenerate, but it can be indefinite). They showed that manifolds of this type are products of an abelian complex group and manifolds which are product of "simple» homogeneous manifolds, (simple here means that they are quotients of simple groups $S$ ).

When these manifolds $M$ satisfy also *-property, then applying the remark on products we obtain the following:

let $M$ be neither compact nor hyperbolic and let's denote by $T$ the product of the non compact factors of $M$ in the above decomposition then, the residual compact product must be hyperbolic. But since a homogeneous compact complex manifold is also complex homogeneous, by Bochner-Montgomery theorem, then $M$ coincides with $T$. Moreover, $T$ must be irreducible, that is, it cannot be biholomorphic with a product of complex manifolds and if it coincides with an abelian group, then it must be one dimensional.

We can conclude, using these remarks and the structure theorem of ([D.Z2]) with:

Let $M$ be a pseudokaehler manifold admitting an effective, transitive and reductive group $G$ of automorphisms and satisfying *-property. Then, we get the following possibility, besides $M$ compact, $C$ or $C^{*}$. 
$M$ is simply connected and $M=S / H$, where $S$ is a simple Lie group, unless $M$ is hyperbolic.

Furthermore, the isotropy subgroup $H$ must be reductive and the complexified group $S^{c}$ contains a parabolic subgroup $P$ such that we get a holomorphic open embedding of $S / H$ as an open $S$-orbit in the rational compact complex flag manifold $S^{c} / P$.

An analogous result occurs when a semisimple Lie group of the first category acts transitively and effectively on the manifold. So, let's first explain this last situation, and then we will see that the case of first category is included in the pseudokaehlerian case.

Let $\mathrm{G}$ be a semisimple Lie group of the first category. A Lie algebra is of the first category if the involutive automorphism generated by a corresponding Cartan decomposition is an inner automorphism (are of this type all the real forms of simple complex Lie algebras with the exception of

$$
S \mathcal{L}(n+1)(n \geqslant 2), \quad S \mathcal{U}^{*}(2 n)(n \geqslant 2), \quad S \mathcal{O}(p, 2 n-p)(p \text { odd }, n \geqslant 4), E I, E I V .
$$

In [M], F. M. Malysev proved that

if $G$ is a semisimple Lie group of the first category and $L$ is a connected closed subgroup of $G$, then $G / L$ has an invariant complex structure iff $G / L$ has even dimension, $L$ is reductive and its semisimple part coincides with the semisimple part of the centralizer of a torus in $G$.

He realizes a holomorphic fibration of $G / L$ over a homogeneous complex manifold $G / L^{*}$, whose complex structure is defined by a parabolic $\mathfrak{p}$ subalgebra of $\mathcal{G}^{c}$ which contains $q$, moreover $G / L^{*}$ is a simply connected domain in a flag manifold. With the hypothesis of *-property we get that $\mathfrak{p}$ must coincide with $q$ since $\mathfrak{p}=\mathcal{N}(\mathfrak{q})=\mathfrak{q}$. So, $G / L$ is already an open $G$-orbit in a flag manifold.

Moreover, by Lemma 6.2 and theorem 6.3 at p. 1150 of ([Wo]) we obtain also that $G / L$ has a $G$-invariant volume element and equivalently, has a $G$-invariant, possibly indefinite, Kaehler metric.

THEOREM 2.3. - Let $M=G / H$ be a homogeneous manifold admitting an effective, transitive and reductive group $G$ and satisfy ${ }^{*}$-property. If one of the following situation occurs $M$ is pseudokaehler, non compact or $G$ is semisimple and of first catego$r y, H$ connected then, $G$ must be simple and $H$ reductive. Moreover $M$ is an open $G$-orbit in a flag manifold and it is simply connected. The case of first category is already pseudokaehler and non compact. Furthermore, if $M$ is not hyperbolic (and then a bounded domain in $C^{n}$ ), then it has no holomorphic functions.

PROOF.- - It remains to prove only the last part. In ([Wo]), p. 1148, J. Wolff associates to an open $S$-orbit a bounded homogeneous domain, which is an irreducible symmetric hermitian space of non compact type: it is the biggest space of this type such that the natural map $S / H \rightarrow S / U$ is $S$-equivariant and holomorphic, here $U$ denotes or 
the maximal compact subgroup of $S$ which contains $H$ either the whole $S$, this last case occurring when the associated hermitian symmetric space reduces to a point.

Now, since $U / H$ is a complex homogeneous manifold, then it cannot lie outside the compact subset given by *-property, unless it has dimension zero. So, only the following cases can occur:

$S / H$ is hyperbolic (and, if this is the case, it is an hermitian symmetric space, that is equivalent to a bounded homogeneous domain of $C^{n}$ );

$S / U$ reduces to a point, and then, see [Wo] pag. 1148, $S / H$ has no non constant holomorphic functions.

In general, about holomorphic functions and *-property, we can say the following:

THEOREM 3.3. - Let G/H have the *-property. Then, either $G / H$ is a bounded domain of $C^{n}$ or it has no non constant bounded holomorphic function.

Proof. - In ([Wi]), Winkelmann defines the hyperbolic reduction of a complex manifold $M$, which is given by the equivalence relation $x=y \leftrightarrow f(x)=f(y)$ for every holomorphic bounded function of $M$. When $M$ is a homogeneous space $G / L$ with an invariant complex structure, then he proves that there is a bounded domain $D$ such that every holomorphic map from $G / L$ to any hyperbolic manifold must fiber through $D$; moreover, the fiber of the reduction is a connected homogeneous complex manifold which is never hyperbolic, unless it is a point (see theorem 1 in [Wi]).

Now, let $G / H$ have the *-property and consider its hyperbolic reduction. Since the fiber is connected and not hyperbolic, then the basis should be compact, and then it must reduce to a point.

\section{REFERENCES}

[Ak1] D. N. AKHIEZER, Lie group actions in complex analysis, Aspects of Math., vol. E, 27 (1995).

[Ak2] D. N. AKHIEZER, Homogeneous complex manifolds, Several complex variables IV, Enc. Math. Sc., vol. 10 (1986), pp. 195-244.

[Ak3] D. N. AHIEZER, Dense orbits with two ends, Math Ussr Izvestija, 11 (1977), pp. 293-307.

[A-H] A. ANDrEotTI - A. HuCKLEBerry, Pseudoconcave Lie groups, Compositio Math., vol. 25, Fasc. 2 (1972).

[Ba-0] W. BARTH - M. OTTE, Über fast-uniform Untergrupen Komplexer Liegruppen und auflosbare Komplexe Mannigfaitigkeiten, Comment. Math. Helv., 44 (1969), pp. 269281.

[Be-O] F. BERTELOOT - K. OELJEKLAUS, Invariant plurisubharmonic functions and hypersurfaces on semisimple complex Lie groups, Math. Ann., 281 (1988) pp. 513-530.

[Bo-M] S. BOCHNER - D. MONTGOMERY, Groups of differentiable and real or complex analytic transformations, Ann. of Math., 46 (1945), pp. 685-694. 
[B-R] A. BOREL - R. REMMERT, Über kompakte homogene komplexe Kaehlersche Mannigfaltigkeiten, Math. Ann., 145 (1962), pp. 429-439.

[D-N] J. DORFMEISTER - K. NAKAJIMA, The fundamental conjecture for homogeneous Kaehler manifolds, Acta Math., 161 (1987), pp. 23-70.

[D-Z1] J. Dorfmeister - ZhuanG-Dan GuAN, Pseudo-Kaehlerian homogeneous spaces admitting a reductive transitive group of automorphisms, Math. Z, 2091 (1992), pp. 89-100.

[D-Z2] J. DoRFMEISTER - ZhUANG-DAN GUAN, Fine structure of reductive Pseudo-Kaehlerian spaces, Geometriae Dedicata, 39 (1991), pp. 321-338.

[Fr] S. FRANKEL, Complex geometry of convex domains that cover varieties, Acta Math., 163 (1989), p. 109-149.

[Fu] H. FujImoto, On the holomorphic automorphism groups of complex spaces, Nagoya, Math. J., Vol. 33 (1968), pp. 85-106.

[G1] B. Gilligan, Ends of homogeneous manifolds, Arch. Math., vol. 37, pp. 544-555.

[G2] B. GiLligav, Holomorphic reductions of homogeneous spaces (Springer) Lect. Notes, Math., 1014 (1983), pag. 27-36.

[G-H1] B. GilligaN - A. HUCKLEBERRY, Complex homogeneous manifolds with two ends, Mich. J. Math., 28 (1981), pp. 183-196.

[G-H2] B. GilligAN - A. HuckLEBERRY, On non-compact complex nilmanifolds, Math. Ann., 238 (1978), pp. 39-49.

[Gr-R] H. GRAUERT - R. REMmERT, Über kompakte homogene komplexe Mannigfaltigkeiten, Arch. Math., 13 (1962), pp. 498-507.

[H] A. T. HUCKLEBERRY, Subvarieties of homogeneous and almost homogeneous manifolds., Aspects Math., 26 (1994).

[Hi] H. HIRONAKA, Introduction to real-analytic sets and real analitic maps, Q.C.N.R. (1973).

[H-01] A. HuCKLEBERRY - E. OELJEKLAUS, Classification theorems for almost homogeneous spaces, Institut E. Cartan, Janvier (1984).

[H-O2] A. HUCKLEBERRY - E. OELJEKLAUS, Homogeneous spaces from a complex analytic viewpoint, Manifolds and Lie groups. Progress in Mathematies, 14 Birkhauser (1981), pp. 159-185.

[H-03] A. T. HuCKLEBERRY - E. OelJEklaus, On holomorphically separable complex solvmanifolds, Ann. Inst. Fourier, 36 (1986), pp. 57-65.

[H-04] A. T. HUCKLEBERRY - E. OELJEKLAUS, A characterization of complex homogeneous cones, Math. Z., 170 (1980) pp. 181-194.

[H-Sn1] A. T. Huckleberry - D. Snow, Pseudoconcave homogeneous manifolds, Ann. Scuola Norm. Sup. Pisa (1980), vol. VII,1, serie IV, pp. 29-54.

[H-Sn2] A. T. HUCKLEBERRY - D. SNOw, A classification of strictly pseudoconcave homogeneous manifolds, Ann. Scuola Norm. Sup. Pisa (1981), pp. 231-255.

[Hp] J. HumphrEYs, Linear algebraic groups, G.T. in Math., vol. 21, Springer-Verlag (1975).

[K1] S. KoBAYASHI, Transformation groups in differential geometry, Springer-Verlag (1972).

[K2] S. KOBAYASHI, Hyperbolic manifolds and holomorphic mappings, Marcel Dekker (1970).

[K-N] S. KOBAYASHI - K. NOMIZU, Foundation of differential geometry, Interscience Publishers (1963).

[Ko] A. KoDAMA, Remarks on homogeneous hyperbolic complex manifolds, Tôhoku Math. Jour., 35 (1983), pp. 181-186.

[M] F. M. MALYSEV, Complex homogeneous spaces of semisimple Lie groups of the first category, Math. USSR Izvestija, vol. 9 (1975), N. 5, pp. 939-949. 
[M-M] Y. Matsushima - A. MoRimoto, Sur certain espaces fibres holomorphes sur une variete de Stein, Bull. Soc. Math. France, 88 (1960) pp. 137-155.

[Ma1] Y. MATSUSHIMA, Espaces homogènes de Stein des groupes de Lie complexes I, Nagoya Math. J., 16 (1960), pp. 205-218.

[Ma2] Y. MATSUShima, Espaces homogènes de Stein des groupes de Lie complexes II, Nagoya Math. J., 18 (1961) pp. 153-164.

[N-S] M. A. NAJMARK - A. I. STERN, Teoria della rappresentazioni dei gruppi, Editori riuniti MIR (1984).

[N] K. NAKAJIMA, Homogeneous hyperbolic manifolds and homogeneous Siegel domains, J. Math. Kyoto Univ., 25-2 (1985), pp. 269-291.

[O-R] K. OElJEKLAUS - W. RICHTHOFER, On the structure of complex solvmanifolds, J. Diff. Geom., 27 (1988)m, pp. 399-421.

[R] R. RICHARDSON, Principal orbit types for reductive groups acting on stein manifolds, Math. Ann., 208 (1974), pp. 323-331.

[Sn1] D. M. SNow, Reductive group actions on Stein spaces, Math. Ann., 259 (1982), pp. 79-97.

[Sn2] D. SNOw, Stein quotients of connected complex lie groups, Manuscripta Math., 50 (1985), pp. 185-214.

[Sn3] D. SNOw, Invariant complex structures on reductive Lie groups, J. Math. B., 371 (1986), pp. 191-215.

[T] J. Tits, Espaces homogènes complexes compacts, Comm. Math. Helv., 37 (1962), pp. 111-120.

[Wa] H. C. WANG, Closed manifolds with homogeneous complex structure, Am. J. Math., 76 (1954), pp. 1-32.

[Wi] J. WinkELMANN, The Kobayashi-pseudodistance on homogeneous manifolds, Manuscripta Math., 68 (1990), pp. 117-134.

[Wo] J. A. WoLF, The action of a real semisimple group on a complex flag manifold, Bull. Amer. Math. Soc., 75 (1969), pp. 1121-1237. 